\title{
The Information-Complete Quantum Theory
}

\author{
Zeng-Bing Chen $\mathbb{D}$ \\ National Laboratory of Solid State Microstructures and School of Physics, Nanjing University, Nanjing 210093, China \\ Correspondence should be addressed to Zeng-Bing Chen; zbchen@nju.edu.cn
}

Received 1 October 2021; Accepted 23 November 2021; Published 31 January 2022

Academic Editor: ShiJie Wei

Copyright (c) 2022 Zeng-Bing Chen. This is an open access article distributed under the Creative Commons Attribution License, which permits unrestricted use, distribution, and reproduction in any medium, provided the original work is properly cited.

Quantum mechanics is a cornerstone of our current understanding of nature and extremely successful in describing physics covering a huge range of scales. However, its interpretation remains controversial since the early days of quantum mechanics. What does a quantum state really mean? Is there any way out of the so-called quantum measurement problem? Here, we present an information-complete quantum theory (ICQT) and the trinary property of nature to beat the above problems. We assume that a quantum system's state provides an information-complete description of the system in the trinary picture. We present a consistent formalism of quantum theory that makes the information-completeness explicitly and argue that conventional quantum mechanics is an approximation of the ICQT. We then show how our ICQT provides a coherent picture and fresh angle of some existing problems in physics. The computational content of our theory is uncovered by defining an information-complete quantum computer.

\section{Introduction}

The unease of understanding quantum theory (QT) began at the very beginning of its establishment. The famous Bohr-Einstein debate $[1,2]$ inspired a lively controversy on quantum foundations. QT is surely an empirically successful theory, with huge applications ranging from subatomic world to cosmology. However, why does it attract such a heated debate over its whole history? The controversial issues on quantum foundations mainly focus on two aspects: (Q1) What does a wave function (or a quantum state) really mean? (Q2) Is the so-called quantum measurement problem [3-9] really a problem? The first axiom of the standard QT states that a system's wave function provides a complete description of the system. But accepting the wave function as QT's central entity, what is the physical meaning of the wave function itself? In this regard, there are two alternatives that the quantum state might be either a state about an experimenter's knowledge or information about some aspects of reality-an epistemic viewpoint-or a state of physical reality-an ontic viewpoint $[10,11]$. A recent result [12] on this issue seems to support the reality of quantum states, yet with ongoing controversy $[13,14]$.
On the contrary, the quantum measurement problem is perhaps the most controversial one on quantum foundations. According to the orthodox interpretation (namely, the Copenhagen interpretation [4]) of QT, a quantum system in a superposition of different states evolves deterministically according to the Schrödinger equation, but actual measurements always collapse, in a truly random way, the system into a definite state, with a probability determined by the probability amplitude according to the Born rule. When, where, and how the quantum state really collapses are out of the reach of QT as it is either "uninteresting or unscientific to discuss reality before measurement" [14].

Our classical world view implies that there exists a world that is objective and independent of any observations. By sharp contrast, what is observed on a quantum system is dependent upon the choice of experimental arrangements; mutually exclusive (or complementary) properties cannot be measured accurately at the same time, a fact known as the complementarity principle. In particular, which type of measurements one would like to choose is totally a free will [15] or a freedom of choice [16-18]. Such a freedom of choice underlies the Pusey-Barrett-Rudolph theorem [12] and the derivation of Bell's inequalities [16-19]. However, one could ask the following: What does a free will or a freedom of 
choice mean physically and particularly, and what is the physical system that encodes information about the free will or freedom of choice?

Thus, in the orthodox interpretation, classical concepts are necessary for the description of measurements (which type of measurements to choose and the particular measurement results for chosen measurement) in QT, although the measurement apparatus can indeed be described quantum mechanically, as done by von Neumann [20, 21]. Seen from its very structure, quantum mechanics "contains classical mechanics as a limiting case, yet at the same time, it requires this limiting case for its own formulation" [22]. In this sense, the current QT has a classical-quantum hybrid feature. At a cosmological scale, the orthodox interpretation rules out the possibility of assigning a wave function to the whole Universe, as no external observer could exist to measure the Universe.

Facing with the interpretational difficulties, various interpretations on QT were proposed by many brilliant thoughts, such as the hidden-variable theory $[16,23]$ (initiated by the famous Einstein-Podolsky-Rosen paper [1] questioning the completeness of QT), many-worlds interpretation $[24,25]$, the relational interpretation $[26,27]$, the decoherence theory [5], and the WISE (wavefunction is the system entity) interpretation $[10,11]$, to mention a few. Thus, "questions concerning the foundations of quantum mechanics have been picked over so thoroughly that little meat is left" [14]. The discovery of Bell's inequalities [19] (as well as the related quantum nonlocality, questioned from the many-worlds interpretation [25]) and the emerging field of quantum information [28] might be among a few exceptions. The recent development of quantum information science sparks the information-theoretical understanding of quantum formalism [29-32].

Logically, if one is looking for a quantum formalism that is universally valid, one should not assume anything beyond the description of the formalism. Current QT does not meet this internal logic requirement as the change of its central entity, quantum states, is separated into two broken pieces-the transition from a probability amplitude (related to a unitary evolution) to a probability (related to a nonunitary measurement) has to require a macroscopic, thus classical, apparatus. Inspired by the classical-quantum hybrid feature of current QT and the abovementioned interpretational progresses, here we present an informationcomplete quantum theory (ICQT) by removing any classical systems/concepts in our description of nature. The ICQT is based on the information-completeness principle: any information must be carried and acquired by certain quantum systems that encode their complete information. In this way, quantum states represent the information-complete code of any possible information that one might acquire. Here, what do we mean by "information" and how to acquire or measure information? In a genuinely quantum world (i.e., there is no room for any classical system), measurement is simply interaction between two physical systems [27]. The interaction creates quantum entanglement between the two systems; what entanglement encodes is the information about the two systems-entanglement between the two systems acquires information of them; no entanglement means no information. Now, it is ready to see that the information-complete physical system must be a composite system, whose constituents interact to mutually acquire information; to be information-complete, the number of the constituents has to be three. As argued below, the three constituents are an indivisible "trinity."

The aim of the present work is thus to suggest a new quantum structure that respects the information-completeness principle. After working out the informationcompleteness explicitly in our formalism, we show that information-complete physical systems, whose definition is to be given below, are characterized by a trinary description, dual entanglement pattern, the emergent dual Born rule, and dual dynamics. As enforced by the information-completeness principle within the trinary picture, an informationcomplete trinity is characterized by the indivisibility of its kinematics and dynamics; its complete information (i.e., the physical predictions) is merely dual entanglement, which acquires a very universal role in our information-complete quantum formalism. The computational content of our theory is uncovered by defining an information-complete quantum computer with a trinary structure and potential of outperforming conventional quantum computers. Seen from the ICQT, the current QT, due to its classical-quantum hybrid feature, is not information-complete and thus suffers from interpretational difficulties.

\section{An Information-Complete Description for Finite-Dimensional Systems}

The orthodox quantum measurement theory [3-5, 7-9] was proposed by von Neumann and can be summarized as follows: for an unknown $d$-dimensional quantum state $|\psi\rangle_{\mathcal{S}}$ of a quantum system $\mathcal{S}$ to be measured, a measurement apparatus ("a pointer") $\mathscr{A}$ is coupled to the system via a unitary operator $\widehat{U}_{\mathcal{S A}}(\widehat{s}, \widehat{p})$. Here, $\widehat{s}$ is system's observable whose eigenstate with respect to the eigenvalue $s_{j}$ reads $|j, \delta\rangle$, namely, $\widehat{s}|j, \mathcal{\delta}\rangle=s_{j}|j, \delta\rangle(j=1,2, \ldots, d) ; \widehat{p}$ is the momentum operator which shifts pointer's $\hat{q}$-reading $([\hat{q}, \hat{p}]=i)$. Assuming that the pointer is initialized in a "ready" state $|0, \mathscr{A}\rangle$ and expanding $|\psi, \mathcal{S}\rangle$ in terms of $|j, \mathcal{S}\rangle$ as $|\psi, \mathcal{S}\rangle=\sum_{j} c_{j}|j, \mathcal{S}\rangle$, then the system and the apparatus are mapped into

$$
\widehat{U}_{\mathcal{S A}}(\widehat{s}, \widehat{p})|\psi, \mathcal{S}\rangle|0, \mathscr{A}\rangle=\sum_{j} c_{j}|j, \mathcal{S}\rangle\left|q_{j}, \mathscr{A}\right\rangle .
$$

To ideally measure $\widehat{s}$, one has to assume that $\mathscr{A}$ must have at least $d$ macroscopically distinguishable pointer positions (plus the ready position corresponding to $|0, \mathscr{A}\rangle$ ), and the pointer state $\left|q_{j}, \mathscr{A}\right\rangle$ and the measured states $|j, \mathcal{S}\rangle$ have an one-to-one correspondence. The above is the usual premeasurement process. The orthodox interpretation of the measurement can only predict the collapse of a definite state $|j, \delta\rangle$ with a probability $\left|c_{j}\right|^{2}$ given by the probability amplitude $c_{j}$; the collapse occurs in a truly random way. For latter convenience, we call $(\widehat{s}, \widehat{p})$ as an observable pair. It is interesting to note that a factorizable structure of the 
"measurement operation" $\widehat{U}_{\mathcal{S A}}(\widehat{\mathcal{S}}, \widehat{p})$ was discovered in the context of the dynamical approach to the quantum measurement problem $[7,8]$.

To avoid the quantum measurement problem, here we take a key step by assuming explicitly information-completeness, whose meaning will be clear below, in our formalism of describing nature. For measuring information on $\mathcal{S}$, one could of course choose various bases, namely, entangle $\mathcal{S}$ and $\mathscr{A}$ in different bases. In the current QT, the observer has the freedom for this kind of choices. However, in the ICQT, we require that all information, including the basis information, must be encoded by certain quantum system to avoid any classical terms or concepts. To this end, starting from a separable state $|\psi, \mathcal{S}\rangle|\phi, \mathscr{A}\rangle$, we introduce

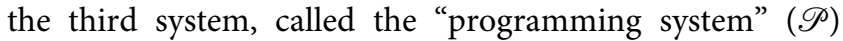
hereafter to encode the basis information of $\mathcal{S}$ and $\mathscr{A}$. We assume that $\mathscr{P}$ has $D_{\mathscr{P}}$ dimensions spanned by $D_{\mathscr{P}}$ orthogonal states, called programming states $|r, \mathscr{P}\rangle$ $\left(r=0,1, \ldots, D_{\mathscr{P}}-1\right)$, where $D_{\mathscr{P}}$ is to be determined by information-completeness. Let us define a unitary programming operation:

$$
\widehat{U}_{\mathscr{P}(\mathscr{S} \mathscr{A})}=\sum_{r=0}^{D_{\mathscr{P}}-1}|r, \mathscr{P}\rangle\langle r, \mathscr{P}| \widehat{U}_{\mathscr{S} \mathscr{A}}\left(\widehat{s}_{r}, \widehat{p}_{r}\right),
$$

which means that if $\mathscr{P}$ is in $|r, \mathscr{P}\rangle$, then do a unitary measurement operation $\widehat{U}_{\mathscr{S A}}\left(\widehat{s}_{r}, \widehat{p}_{r}\right)$ on $\mathcal{S} \mathscr{A}$. Now suppose that $\mathscr{P}$ is prepared in an initial state $|\chi, \mathscr{P}\rangle=\sum g_{r}|r, \mathscr{P}\rangle$. Then the state of the whole system $\mathscr{P} \mathscr{S} \mathscr{A}$ after the programming operation reads

$$
|\mathscr{P}(\mathcal{S} \mathscr{A})\rangle=\sum_{r=0}^{D_{\mathscr{P}}-1} g_{r}|r, \mathscr{P}\rangle|r, \mathcal{S} \mathscr{A}\rangle,
$$

where $|r, \mathcal{S A}\rangle=\widehat{U}_{\mathcal{S A}}\left(\widehat{s}_{r}, \widehat{p}_{r}\right)|\psi, \mathcal{S}\rangle|\phi, \mathscr{A}\rangle$ encodes the programmed entanglement, if any, between $\mathcal{S}$ and $\mathscr{A}$. For a given $|r, \mathscr{P}\rangle$, the pair observables (denoted by $\left(\widehat{s}_{r}, \widehat{p}_{r}\right)$ ) and their information to be measured are determined by the Schmidt form of $|r, \mathcal{S} \mathscr{A}\rangle$. Note that $|\mathscr{P}(\mathscr{S} \mathscr{A})\rangle$ can also be written in a Schmidt form with positive real coefficients [33]. Hereafter we suppose that $|\mathscr{P}(\mathscr{S} \mathscr{A})\rangle$ has been Schmidtdecomposed and $g_{r}>0$ (in practice, we could of course have the situation where $\mathscr{P}$ and $\mathcal{S} \mathscr{A}$ are not fully entangled, i.e., the number of $g_{r}>0$ is less than $D_{\mathscr{P}}$ or $d D_{\mathscr{A}}$ ).

Now the key point of our formalism is to require that the programming system $\mathscr{P}$ encodes all possible, namely, information-complete, measurement operations that are allowed to act upon the $\mathcal{S} \mathscr{A}$-system. To be "informationcomplete," all programmed measurement operations $\widehat{U}_{\mathcal{S A}}\left(\widehat{s}_{r}, \widehat{p}_{r}\right)$ can at least achieve the measurements of a complete set of operators for $\delta$; for the $d$-dimensional system, the complete set has $d^{2}$ operators [34], i.e., the minimal $D_{\mathscr{P}}=d^{2}$. Note that the information-complete set of operators or measurement is also important for quantum state tomography $[35,36]$.

Another trick in the above discussion is that, to enable the information-complete programmed measurements, it seems that one needs $D_{\mathscr{P}}$ different measurement apparatuses. Hereafter we take a step further by dropping this specific measurement model by regarding the $\mathscr{A}$-system as a single quantum system (not necessarily having $\widehat{p}$ and $\widehat{q}$ as in the specific model that we considered above) with $D_{\mathscr{A}}(\geq d)$ dimensions, and as such, the standard premeasurement process described above is simply to entangle $\mathcal{S}$ and $\mathscr{A}$ (see the next section for further discussion). In this case, we have $D_{\mathscr{P}} \geq d D_{\mathscr{A}}$ due to the property of the Schmidt decomposition. The step is necessary for seeking a model-independent and intrinsic description of the whole system $\mathscr{P} \mathcal{S} \mathscr{A}$.

To have an easy understanding of our informationcomplete description of physical systems, some remarks are necessary. First, we note that the third system is also included in other interpretations of QT, such as the manyworlds interpretation $[24,25]$, the relational interpretation $[26,27]$, and the "objective quantum measurement" [37]. However, the third system in our formalism plays a role that is dramatically different from those interpretations. Actually, imposing information-completeness into our quantum description of nature distinguishes our theory from all the previous interpretations of QT. Second, the fact that $|r, \mathcal{S A}\rangle$, as entangled, can always be written in a Schmidt form implies a symmetric role played by $\mathcal{S}$ and $\mathscr{A}$. Meanwhile, the role of $\mathscr{P}$ is dramatically different from that of either $\mathcal{S}$ or $\mathscr{A}$. But $\mathscr{P}$ and the combined system $\mathcal{S} \mathscr{A}$ play a symmetric role. We anticipate that such a feature could have profound consequences, particularly for the internal consistency of the theory. We will find that this is indeed the case when we consider the dynamics within the ICQT.

\section{The Emergent Dual Born Rule}

How to acquire information and which kind of information to acquire are two questions of paramount importance. In usual quantum measurement problem [6], the problem of definite outcomes and the problem of the preferred basis answer the two problems separately. In particular, the existence of a preferred basis gives an explanation for why a specific pointer basis gets chosen and, ultimately, for the appearance of classicality. However, we should keep in mind that the Schmidt basis, as we used in the ICQT, does not necessarily correspond to a preferred basis, whose existence requires other criterion for classicality.

According to the ICQT, on one hand, the only way to acquire information is to interact (i.e., entangle) the system $\mathcal{S}$ and the apparatus $\mathscr{A}$ with each other; no interaction leads to no entanglement and thus no information. This is in a similar spirit as the relational interpretation $[26,27]$, which treats the quantum state as being observer-dependent, namely, the state is the relation between the observer and the system. On the other hand, the programming system $\mathscr{P}$, by interacting with $\mathcal{S A}$, dictates the way (actually, the information-complete way) on which kind of information (called the " $\mathscr{P}-\mathcal{S} \mathscr{A}$ information" hereafter) to acquire about the system $\mathcal{S}$. For instance, if the whole system is programmed to measure $\widehat{s}_{r}$, then $\delta$ and $\mathscr{A}$ interact with each other to induce the programmed measurement operations $\widehat{U}_{\mathscr{S A}}\left(\widehat{s}_{r}, \widehat{p}_{r}\right)$. This process generates the entangled state $|r, \mathcal{S} \mathscr{A}\rangle$ with which $\mathscr{A}$ "knows," in a completely coherent way, all information (called the "programmed $\left.\mathscr{S} \mathscr{A}\right|_{\mathscr{P}}$ 
information") about $\mathcal{S}$ in the basis of $\widehat{s}_{r}$; the amount of entanglement contained in $|r, \mathcal{S} \mathscr{A}\rangle$ quantifies the amount of information acquired during this measurement. Also, $\mathscr{P}$ "knows," again in a completely coherent way, the information about which kind of information (here $|r, \mathcal{S} \mathscr{A}\rangle) \mathscr{A}$ has about $\mathcal{S}$; the amount of the $\mathscr{P}_{-}(\mathcal{S} \mathscr{A})$ entanglement quantifies the amount of information on which kind of measurements to do. All information is coherently and completely encoded there by certain quantum system.

Thus, for any given system $\mathcal{S}$ in our description, one has to ask two questions: how $\mathcal{S}$ gets entangled with another measurement system $\mathscr{A}$ and how many independent ways can it be entangled with $\mathscr{A}$ ? The answer to the latter question is completely contained in the entangled state $|\mathscr{P}(\mathcal{S} \mathscr{A})\rangle$, while the answer to the former is the programmed entanglement $|r, \mathcal{S} \mathscr{A}\rangle$-the two questions are answered by entanglement at two different levels, called dual entanglement.

Now let us state a key point in our ICQT. Namely, entanglement (created by interaction), necessary and sufficient for acquiring information, is the measurement and the physical predictions of the theory as any possible information (the $\mathscr{P}-\mathcal{S} \mathscr{A}$ information and the programmed $\left.\mathcal{S} \mathscr{A}\right|_{\mathscr{P}}$ information) is completely encoded in the particular dual entanglement structure of the whole system. To see this, let us note that the reduced density operators for $\mathscr{P}$ and $\mathcal{S} \mathscr{A}$ read

$$
\begin{gathered}
\rho_{\mathscr{P}}=\operatorname{tr}_{\mathscr{S} \mathscr{A}}\left[|\mathscr{P}(\mathcal{S} \mathscr{A})\rangle\langle\mathscr{P}(\mathcal{S} \mathscr{A}) \|]=\sum_{r=0}^{D_{\mathscr{P}}-1} g_{r}^{2}|r, \mathscr{P}\rangle\langle r, \mathscr{P}|,\right. \\
\rho_{\mathscr{S} \mathscr{A}}=\operatorname{tr}_{\mathscr{P}}\left[|\mathscr{P}(\mathcal{S} \mathscr{A})\rangle\left\langle\mathscr{P}(\mathcal{S} \mathscr{A}) \|=\sum_{r=0}^{D_{\mathscr{P}}-1} g_{r}^{2} \mid r, \mathcal{S} \mathscr{A}\right\rangle\langle r, \mathcal{S} \mathscr{A}|,\right.
\end{gathered}
$$

implying that all information about $\mathscr{P}(\mathscr{S} \mathscr{A})$ is completely contained in the set $\left\{g_{r}^{2},|r, \mathscr{P}\rangle\right\}\left(\left\{g_{r}^{2},|r, \mathcal{S} \mathscr{A}\rangle\right\}\right)$, the physical predictions of the theory. Yet, all these physical predictions are already encoded completely in the $\mathscr{P}_{-}(\mathcal{S} \mathscr{A})$ entanglement. In other words, the $\mathscr{P}-(\mathcal{S A})$ entanglement is sufficient to predict $\left\{g_{r}^{2},|r, \mathscr{P}\rangle\right\}$ and $\left\{g_{r}^{2},|r, \mathcal{S} \mathscr{A}\rangle\right\}$, a task that we could expect for a measurement. Similar analysis applies to the programmed entanglement $|r, \mathcal{S} \mathscr{A}\rangle$ as well.

As both $|r, \mathcal{S} \mathscr{A}\rangle$ and $|\mathscr{P}(\mathcal{S} \mathscr{A})\rangle$ are pure states, entanglement for each of them is uniquely quantified by the usual entanglement entropy $[33,38]$. Here, the $\mathscr{P}-(\mathcal{S} \mathscr{A})$ entanglement-a two-party entanglement, although having three constituents $(\mathscr{P}, \mathscr{A}$, and $\mathcal{S}$, called the trinity)quantifies the $\mathscr{P}-\mathcal{S} \mathscr{A}$ information and has entanglement entropy maximally $\ln D_{\mathscr{P}}$, while the $\mathcal{S}$ - $\mathscr{A}$ entanglement contained in $|r, \mathcal{S} \mathscr{A}\rangle$ quantifies the programmed $\left.\mathcal{S} \mathscr{A}\right|_{\mathscr{P}}$ information and has entanglement entropy maximally $\ln D_{\mathcal{S}}$. This immediately identifies each of the squared coefficients of their Schmidt decompositions as a probability [33] to reconcile with Shannon's definition of entropy. Put differently, in our information-complete description of physical systems, entanglement does be all the information; classical terms like probability arise in our description because of either our reliance on classical concept of information or certain approximate and incomplete description to be shown below. By regarding entanglement directly as measurement of complete information, one can avoid the classical-quantum hybrid feature of current quantum theory or any classical concepts having to use therein. Namely, one does not need to ask nature classical questions via the conventional measurement postulate if one believes that nature is fundamentally quantum mechanical. Of course, the measurement postulate is absolutely needed for the conventional formulation of QT to make physical predictions. By contrast, physical predictions in the ICQT are completely encoded by entanglement of the trinity.

The status of quantum states (more precisely, dual entanglement) in the information-completeness formalism thus represents a complete reality of the whole system $(\mathscr{P}, \mathcal{S}$, and $\mathscr{A}$, the trinity). Such a reality picture ("quantum reality") is only possible by taking into account the informationcompleteness explicitly in our formalism. Quantum states do exist in a world that is informational and objective. Whatever an observation might be, information-complete states always encode information pertaining to that observation as programmed, without invoking observers or having to appeal to any mysterious mechanisms to account for wave function collapse; there is simply no wave function collapse. Here local quantum states (i.e., states for each of $\mathscr{P}$, $\mathcal{S}$, and $\mathscr{A}$ ) are all relative, but information encoded in dual entanglement is invariant under the changes of local bases, a basic property of entanglement. If one likes, the choice of local bases can be called a free will or freedom of choice, corresponding to certain "gauge." Yet, all physical predictions of the theory are encoded in dual entanglement and do not depend on the chosen gauge. To compare with the relational interpretation $[26,27]$, the ICQT tells us a kind of quantum relationalism-while local quantum states of a single system are merely of relative meaning, the relations between states of two systems have physical significance; in a truly quantum world, entanglement is the relations.

In certain sense, $\mathscr{P}$ and $\mathscr{A}$ act like a "quantum being" ("qubeing") who holds coherently all the informationcomplete programmes on how to entangle $\mathcal{S}$ and $\mathscr{A}$. In this way, the qubeing has all the information about $\mathcal{S}$. However, our human beings plus the measurement apparatuses, unlike the qubeing, are macroscopic and have so many quantum degrees of freedom. For example, an experimenter, Alice, together with her apparatus, would like to acquire information about $|\psi, \mathcal{S}\rangle$. First of all, she has to decide which kind of information she would like to know. After making a decision, she needs then to observe (that is, to interact with) her apparatus readily entangled with $\mathcal{\delta}$. In principle, Alice's decisions and observations are all physical processes which should be described quantum mechanically. Thus, if the total system is isolated, it must be in dual entanglement, encoding complete information of the whole system, as argued above. Nevertheless, Alice, a human being, is so used to and familiar with classical concepts on information and physical systems. Practically, she has limited ability and is lack of full knowledge of the entire system. In this case, she has to "trace out" those quantum degrees of freedom involved in her decisions (interaction between $\mathscr{P}$ and $\mathcal{S A})$, leading to a mixed state $\sum_{r}\left|g_{r}\right|^{2}|r, \mathscr{P}\rangle t \mid\langle r, \mathscr{P}||$ 
and $\sum_{r}\left|g_{r}\right|^{2}|r, \mathcal{S} \mathscr{A}\rangle t \mid\langle r, \mathcal{S} \mathscr{A} \|$ (see equation (4)). This state allows a probability interpretation about Alice's freedom of choice: each of her decisions $|r, \mathscr{P}\rangle$ occurs with a probability of $\left|g_{r}\right|^{2}$. As far as a particular choice $|r, \mathscr{P}\rangle$ has been made, again she has to trace out her quantum degrees of freedom involved in her observation (interaction between $\mathscr{A}$ and $\mathcal{S}$ ). This then leads to the usual Born rule about $|\psi, \mathcal{S}\rangle$ for the given measurement. Thus, in the ICQT, the Born rule, also in dual form, is an emergent or derived rule determined by the dual entanglement structure.

To summarize the above picture, the world view of the ICQT is fascinating. If we regard the system $\delta$ as an indivisible part of the qubeing $\mathscr{P} \mathscr{A}$, the whole system $\mathscr{P} \mathscr{S} \mathscr{A}$ then represents an information-complete and objective entity; it seems that the qubeing has its own "consciousness," a kind of miraculous quantum ability, to encode and access all its information in the form of dual entanglement, in which the constituent parts of the qubeing are mutually measured or defined. In other words, for the qubeing, all information (namely, all physical predictions) is encoded in dual entanglement via interaction, but not obtained via the usual quantum measurement with the unavoidable concept of the wave function collapse.

The trinary picture (the division of $\mathcal{S}, \mathscr{A}$, and $\mathscr{P}$ ) of physical systems arises here as a new feature of the ICQT, as shown in Figure 1. To retain the information-complete description of nature, such a trinary picture seems to be unavoidable. The limitation of information contents in dual entanglement could be tentatively called "the trinity principle," instead of the conventional complementarity principle, to put the trinary property of physical systems on the most fundamental ground.

The loss of the trinary picture of describing physical systems leads to the emergent dual Born rule, i.e., the probability description on which kind of observables to measure and then on which eigenvalue of the observable to measure, due to, e.g., lack of full knowledge of the entire system in our ICQT. The conventional von Neumann entropy quantifies this dual loss of information. In other words, the conventional Born rule arises as a consequence of the sacrifice of information-complete description in the trinary picture; the sacrifice leads to a partial reality of physical system as described by conventional QT.

\section{The Information-Complete Dynamics}

According to the above picture of nature, single free systems are simply meaningless for acquiring information; a system, which does not give information to (i.e., interact with) other systems in any way, simply does not exist. The " $\mathcal{S}+\mathscr{A}$ " description in the usual QT is also inadequate because of its information-incompleteness as there is certain information (e.g., the choice of measurement bases) not encoded by any physical quantum system. Therefore, the dynamics of the ICQT will be dramatically different from the usual picture as it requires interacting $\mathscr{P}+\mathscr{S} \mathscr{A}$ so as to obey the information-completeness principle within the trinary picture. Without specifying the $\mathscr{P}+\mathcal{S} \mathscr{A}$ dynamics to maintain the

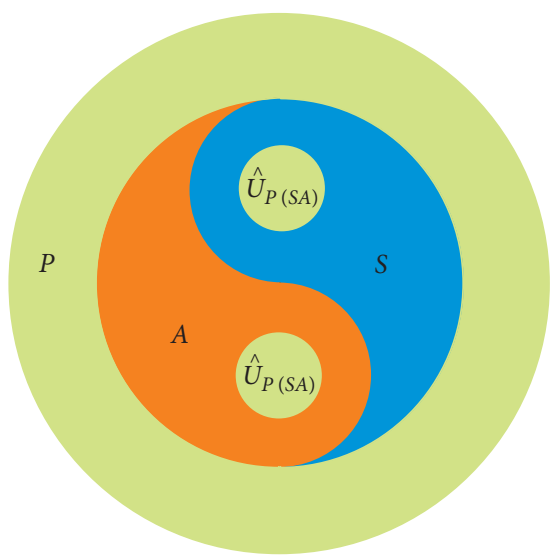

FIgURE 1: The trinary picture of the world. The division of system $\mathcal{S}$, measurement apparatus $\mathscr{A}$, and programming system $\mathscr{P}$ naturally arises in the information-complete description of physical systems. "The Taiji pattern" shows in an intuitive manner the $\mathcal{S}-\mathscr{A}$ interaction (entanglement), while the green discs inside and outside the Taiji pattern represent the programmed measurement operations $\widehat{U}_{\mathscr{P}(\mathcal{S} \mathscr{A})}$ between $\mathscr{P}$ and $\mathcal{S} \mathscr{A}$. In ancient China, Taoists regarded the Taiji pattern as a "diagram of the Universe." The trinary picture of the world shown here is ubiquitous in the sense that the world, at the most fundamental level, is made up of a trinity: gravity (i.e., spacetime, $\mathscr{P}$ ), elementary matter fermions $(\mathcal{S})$, and their gauge fields $(\mathscr{A})$; the trinity should be describable by the ICQT.

information-complete trinary description, it is meaningless or information-incomplete to specify local states of single systems in $\mathscr{P}+\mathcal{S} \mathscr{A}$. Namely, the ICQT is characterized by the indivisibility of its kinematics and dynamics. Below, we give some key features of the information-complete dynamics.

Before considering the information-complete dynamics, let us introduce an important concept of dual measurability: the $\mathscr{P}-\mathcal{S} \mathscr{A}$ measurability and the programmed $\left.\delta \mathscr{A}\right|_{\mathscr{P}}$ measurability. The former means the ability of measuring $\mathscr{P}$ with $\mathcal{S A}$ and vice versa; the latter means the ability of measuring $\mathscr{A}$ with $\delta$ and vice versa, under a given programmed measurement operation of $\mathscr{P}$. The $\mathscr{P}-\mathcal{S} \mathscr{A}$ measurability (the programmed measurability $\left.\mathcal{S} \mathscr{A}\right|_{\mathscr{P}}$ ) leads to $D_{\mathscr{P}}=D_{\mathscr{A}} D_{\mathscr{S}}\left(D_{\mathscr{A}}=D_{\mathcal{S}}=D\right)$ and thus a symmetric role between $\mathscr{P}$ and $\mathscr{S} \mathscr{A}(\mathcal{S}$ and $\mathscr{A})$. Note that here measurability does not mean certain quantum measurement actually performed by an experimenter in the usual sense. As we pointed out above, in the ICQT, entanglement is the measurement. Thus, dual measurability is simply another side of dual entanglement; the relation among the dimensions for the trinity's quantum states is then a simple consequence of the Schmidt decompositions of dual entanglement, which is the complete physical prediction of our theory.

After the above preparation, now we give a definition of information-complete physical systems: a physical system is said to be information-complete if and only if (the use of "if and only if" will be explained below) it is consisted of $\mathcal{S}, \mathscr{A}$, and $\mathscr{P}$ described as a trinity such that the $\mathscr{P}-\mathcal{S} \mathscr{A}$ measurability and the programmed measurability $\left.\mathcal{S} \mathscr{A}\right|_{\mathscr{P}}$ are satisfied. As a result of this definition, the $\mathscr{P}-\mathcal{S} \mathscr{A}$ 
measurability implies the existence of at most $D^{2}$ independent information-complete measurement (entanglement) operations in the Hilbert spaces of both $\mathscr{P}$ and $\mathcal{S A}$; these operations generate at most $D^{2}$-dimensional entanglement between $\mathscr{P}$ and $\mathscr{S} \mathscr{A}$ and at most $D^{2}$ entangled states between $\mathcal{S}$ and $\mathscr{A}$ such that $\mathscr{P}$ and $\mathcal{S} \mathscr{A}$ are mutually measuring and defining. Meanwhile, under the given programming state of $\mathscr{P}$, the programmed measurability $\left.\mathcal{S} \mathscr{A}\right|_{\mathscr{P}}$ implies the existence of at most $D$-dimensional entangled states between $\mathcal{S}$ and $\mathscr{A}$ such that $\mathcal{S}$ and $\mathscr{A}$ are mutually measuring and defining, as programmed. For convenience, we also call the complete set of states and operators defined in the Hilbert space of $\mathscr{P}$ or $\mathcal{S A}$ information-complete. Accordingly, states and operators for either $\mathcal{S}$ or $\mathscr{A}$ alone are information-incomplete. Thus, in the ICQT, the role of observables defined for either $\delta$ or $\mathscr{A}$ is quite different from the role of observables defined for $\mathscr{P}$.

Let us suppose that the information-complete system $\mathscr{P} \mathcal{S} \mathscr{A}$ has a general Hamiltonian $\widehat{H}_{\mathscr{P} \mathcal{S} \mathscr{A}}$. We assume that the whole system evaluates according to the standard Schrödinger equation (we take $\hbar=1$ ), namely, $i(\mathrm{~d} / \mathrm{d} t)|\mathscr{P}(\mathcal{S A}), t\rangle=\widehat{H}_{\mathscr{P} \mathcal{S} \mathscr{A}}|\mathscr{P} \mathcal{S} \mathscr{A}, t\rangle$. In general, $\widehat{H}_{\mathscr{P} \mathcal{S} \mathscr{A}}=$ $\widehat{H}_{\mathscr{P}}+\widehat{H}_{\mathcal{S}}+\widehat{H}_{\mathscr{A}}+\widehat{H}_{\mathscr{P} \mathcal{S}}+\widehat{H}_{\mathscr{P} \mathscr{A}}+\widehat{H}_{\mathscr{S} \mathscr{A}}+\widehat{H}_{\mathscr{P}(\mathscr{S} \mathcal{A})}$, where the subscripts label the corresponding systems. Now our problem is to determine how the information-completeness principle constrains the form of $\hat{H}_{\mathscr{P} \mathcal{S} \mathscr{A}}$ and thus the dynamics of the $\mathscr{P} \mathscr{S} \mathscr{A}$-system.

Note that we can choose an orthonormal basis (the "programming basis") $\left\{\left|e_{n}, \mathscr{P}\right\rangle ; n=0,1, \ldots, D_{\mathscr{P}}-1\right\}$ to span the whole Hilbert space of $\mathscr{P}$. We associate each programming state $\left|e_{n}, \mathscr{P}\right\rangle$ as an eigenstate of $\mathscr{P}$-system's "programming observable" $\hat{e}_{\mathscr{P}}$ with eigenvalue $e_{n}$. It is easy to verify that the following Hamiltonian obeys the informational completeness principle:

$$
\begin{aligned}
\hat{\mathscr{I}}_{\mathscr{P} \mathcal{S} \mathscr{A}}= & \widehat{H}_{\mathscr{P}}+\widehat{H}_{\mathscr{P}(\mathcal{S A})}=\widehat{H}_{\mathscr{P}} \\
& +\sum_{n=0}^{D_{\mathscr{P}}-1}\left|e_{n}, \mathscr{P}\right\rangle\left\langle e_{n}, \mathscr{P}\right| \widehat{H}_{\left.\mathcal{S} \mathscr{A}\right|_{\mathscr{P}}}\left(e_{n}, t\right),
\end{aligned}
$$

if we impose the $\mathscr{P}-\mathcal{S} \mathscr{A}$ measurability condition as follows:

$$
\left[\widehat{H}_{\mathscr{P}(\mathcal{S} \mathscr{A})}, \widehat{e}_{\mathscr{P}}\right]=\left[\widehat{H}_{\mathscr{P}}, \widehat{e}_{\mathscr{P}}\right]=0 \text {, }
$$

namely, $\hat{e}_{\mathscr{P}}$ commutes with both $\hat{H}_{\mathscr{P}(\mathscr{S A})}$ and $\hat{H}_{\mathscr{P}}$. If one takes $\widehat{e}_{\mathscr{P}}=\widehat{H}_{\mathscr{P}}$, equation (6) reads simply as

$$
\left[\widehat{H}_{\mathscr{P}(\mathscr{S A})}, \widehat{H}_{\mathscr{P}}\right]=0 .
$$

In equation (5), $\widehat{H}_{\left.\mathcal{S} \mathscr{A}\right|_{\mathscr{P}}}\left(e_{n}, t\right)=i(\mathrm{~d} / \mathrm{d} t) \widehat{U}_{\mathcal{S A} \mid \mathscr{P}}\left(e_{n}, t\right)$. $\widehat{U}_{\left.\mathcal{S} \mathscr{A}\right|_{\mathscr{P}}}^{-1}\left(e_{n}, t\right)$ is an information-complete operator set of $\mathcal{S} \mathscr{A} ; \sum_{n=0}^{D_{\mathscr{P}}-1}\left|e_{n}, \mathscr{P}\right\rangle\left\langle e_{n}, \mathscr{P}\right| \widehat{H}_{\left.\mathscr{S} \mathscr{A}\right|_{\mathscr{A}}}\left(e_{n}, t\right)$ represents actually the spectrum-decomposition with respect to $\hat{e}_{\mathscr{P}}$ of $\widehat{H}_{\mathscr{P}(\mathcal{S} \mathscr{A})}\left(\widehat{e}_{\mathscr{P}}, t\right)$. Meanwhile, $\widehat{\mathscr{I}}_{\mathscr{P} \mathcal{S} \mathscr{A}}$ takes a form like $\hat{\mathscr{I}}_{\mathscr{P} \mathcal{S} \mathscr{A}}=\hat{H}_{\mathscr{P}}+\widehat{H}_{\mathscr{P} \mathcal{S}}+\widehat{H}_{\mathscr{P} \mathscr{A}}+\widehat{H}_{\mathscr{P}(\mathcal{S} \mathscr{A})}$ such that $\mathscr{P}$ universally couples with $\mathcal{S}$ and $\mathscr{A}$. Note that in $\hat{\mathscr{I}}_{\mathscr{P} \mathcal{S} \mathscr{A}}$, Hamiltonians $\widehat{H}_{\delta}, \widehat{H}_{\mathscr{A}}$, and $\widehat{H}_{\mathscr{H}}$ do not appear. These "local" Hamiltonians $\left(\widehat{H}_{\mathcal{S}}, \widehat{H}_{\mathscr{A}}\right.$, and $\left.\widehat{H}_{\mathcal{S}}\right)$ decoupled from $\mathscr{P}$ induce local unitary transformations upon $\mathcal{S}$ or/ and $\mathscr{A}$, corresponding to certain gauge, which can be gauged out in physical predictions encoded in dual entanglement.

$\hat{\mathscr{I}}_{\mathscr{P} \mathcal{S} \mathscr{A}} \quad$ induces an evolution $|\mathscr{P}(\mathcal{S} \mathscr{A}), t\rangle=\widehat{U}_{\mathscr{P} \mathcal{S} \mathscr{A}}|\mathscr{P}, t=0\rangle|\mathcal{S} \mathscr{A}, t=0\rangle$; the evolution operator $\widehat{U}_{\mathscr{P} \mathcal{S} \mathscr{A}}$ always has a factorizable structure:

$$
\widehat{U}_{\mathscr{P} \mathcal{S} \mathscr{A}}(t)=\sum_{n=0}^{D_{\mathscr{P}}-1}\left|e_{n}, \mathscr{P}\right\rangle\left\langle e_{n}, \mathscr{P}\right| \widehat{U}_{\mathscr{P}}(t) \widehat{U}_{\left.\mathcal{S} \mathscr{A}\right|_{\mathscr{P}}}\left(e_{n}, t\right),
$$

as a result of the $\mathscr{P}-\mathcal{S} \mathscr{A}$ measurability condition (6), such that $\left(\forall e_{n}\right)$

$$
\begin{aligned}
i \frac{\mathrm{d}}{\mathrm{d} t} \widehat{U}_{\mathscr{P}}(t) & =\widehat{H}_{\mathscr{P}} \widehat{U}_{\mathscr{P}}(t), \\
i \frac{\mathrm{d}}{\mathrm{d} t} \widehat{U}_{\left.\mathcal{S} \mathscr{A}\right|_{\mathscr{P}}}\left(e_{n}, t\right) & =\widehat{H}_{\left.\mathscr{S} \mathscr{A}\right|_{\mathscr{P}}}\left(e_{n}, t\right) \widehat{U}_{\left.\mathscr{S} \mathscr{A}\right|_{\mathscr{P}}}\left(e_{n}, t\right) .
\end{aligned}
$$

In this way, the dynamical evolutions of $\mathscr{P}$ and $\mathcal{S} \mathscr{A}$ are mutually defined, in accordance with the informationcompleteness principle.

The Hamiltonian $\hat{\mathscr{I}}_{\mathscr{P} \mathcal{S} \mathscr{A}}$ as given above respects the $\mathscr{P}-\mathcal{S} \mathscr{A}$ measurability. If we also require the programmed measurability $\left.\mathcal{S} \mathscr{A}\right|_{\mathscr{P}}\left(\forall e_{n}\right)$, the evolution governed by $\widehat{H}_{\mathcal{S} \mathscr{A} \mid \mathscr{P}}\left(e_{n}, t\right)$ depends on which system $(\mathcal{S}$ or $\mathscr{A})$ defines the programming observable. For example, if one can find the programming observable $\widehat{\mathcal{E}}_{\left.\mathcal{S}\right|_{\mathscr{P}}}$ for $\mathcal{S}$, the programmed evolution for $\mathscr{S} \mathscr{A}$ will similarly acquire the factorizable structure as

$$
\begin{aligned}
\widehat{H}_{\left.\mathcal{S A}\right|_{\mathscr{P}}}\left(e_{n}, t\right)= & \sum_{i=0}^{D-1}\left|\varepsilon_{i}\left(e_{n}\right), e_{n}, \delta\right\rangle\left\langle\varepsilon_{i}\left(e_{n}\right), e_{n}, \delta\right| \\
& \times \widehat{H}_{\mathscr{A}_{|s|} \mid}\left[e_{n}, \varepsilon_{i}\left(e_{n}\right), t\right] \\
& +\widehat{H}_{\left.\mathcal{S}\right|_{\mathscr{P}}}\left(e_{n}, t\right)+\widehat{H}_{\left.\mathscr{A}\right|_{\mathscr{P}}}\left(e_{n}, t\right),
\end{aligned}
$$

with $\quad \widehat{H}_{\left.\mathscr{A}_{||}\right|_{\mathscr{P}}} \equiv i(\mathrm{~d} / \mathrm{d} t) \widehat{U}_{\left.\mathscr{A}_{\mid s}\right|_{\mathscr{P}}}\left[e_{n}, \varepsilon_{i}\left(e_{n}\right), t\right] \cdot \widehat{U}_{\left.\mathscr{A}_{|S|}\right|_{\mathscr{S}}}^{-1}\left[e_{n}, \varepsilon_{i}\right.$ $\left.\left(e_{n}\right), t\right]$. Here the orthonormal basis for $\left.\delta\right|_{\mathscr{P}}$ is $\left\{\left|\varepsilon_{i}\left(e_{n}\right), e_{n}, \mathcal{S}\right\rangle\right\}$, where $\left|\varepsilon_{i}\left(e_{n}\right), e_{n}, \mathcal{S}\right\rangle$ is an eigenstate of $\widehat{\mathcal{E}}_{\mathcal{S} \mid \mathcal{P}}\left(e_{n}\right)$ with eigenvalue $\varepsilon_{i}\left(e_{n}\right)$ for given $e_{n}$. Similarly to the $\mathscr{P}-\mathcal{S} \mathscr{A}$ measurability condition (6), we need to impose the programmed measurability $\left.\mathcal{S} \mathscr{A}\right|_{\mathscr{P}}$ condition $\left(\forall e_{n}\right)$ :

$$
\left[\widehat{H}_{\left.\mathcal{S} \mathscr{A}\right|_{\mathscr{P}}}\left(e_{n}, t\right), \widehat{\varepsilon}_{\left.\mathcal{S}\right|_{\mathscr{P}}}\left(e_{n}\right)\right]=\left[\widehat{\mathcal{E}}_{\left.\mathcal{S}\right|_{\mathscr{P}}}\left(e_{n}\right), \widehat{H}_{\mathcal{S}_{\mid \mathscr{P}}}\left(e_{n}, t\right)\right]=0 \text {. }
$$

The $\left.\mathscr{S} \mathscr{A}\right|_{\mathscr{P}}$ dynamics is then similar to the $\mathscr{P}-\mathcal{S} \mathscr{A}$ dynamics considered above. Such a dual dynamics of the whole system $\mathscr{P} \mathscr{S} \mathscr{A}$ is an attribute of the trinary description and quite distinct to the usual Schrödinger evolution.

What is the physical significance of the programming basis $\left\{\left|e_{n}, \mathscr{P}\right\rangle\right\}$ and the associated observable $\hat{e}_{\mathscr{P}}$ ? Actually it is physically transparent that $\left\{\left|e_{n}, \mathscr{P}\right\rangle\right\}$ as the physical predictions can be identified with the Schmidt basis for the $\mathscr{P}-\mathcal{S} \mathscr{A}$ decomposition, which is not affected by the local transformations generated by $\widehat{H}_{\mathscr{P}}$. A more interesting possibility is to interpret $\widehat{e}_{\mathscr{P}}$ as a quantum nondemolition observable [39, 40]; note that the premeasurement involves a nondemolition coupling between $\delta$ and $\mathscr{A}$ [37]. Then the $\mathscr{P}$ - $\mathcal{S} \mathscr{A}$ measurability condition (6) is a (sufficient) condition 
for a quantum nondemolition measurement of $\widehat{e}_{\mathscr{P}}$. In the context of quantum nondemolition observable, a more general measurability condition could be imposed. For instance, the $\mathscr{P}-\mathcal{S} \mathscr{A}$ measurability condition in equation (6) might be replaced by

$$
\left[\widehat{U}_{\mathscr{P}}(t), \widehat{e}_{\mathscr{P}}\right]|\mathscr{P}(\mathcal{S} \mathscr{A}), t\rangle=\left[\widehat{U}_{\mathscr{P} \mathcal{S} \mathscr{A}}(t), \widehat{e}_{\mathscr{P}}\right]|\mathscr{P}(\mathcal{S} \mathscr{A}), t\rangle=0 .
$$

As we require that the evolution $|\mathscr{P}(\mathcal{S} \mathscr{A}), t\rangle=\widehat{U}_{\mathscr{P} \mathcal{S} \mathscr{A}}|\mathscr{P}, t=0\rangle|\mathcal{S} \mathscr{A}, t=0\rangle$ results in a state already in the Schmidt form

$$
\begin{aligned}
|\mathscr{P}(\mathcal{S} \mathscr{A}), t\rangle & =\sum_{r=0}^{D_{\mathscr{P}}-1} g_{r}\left|e_{n}, \mathscr{P}\right\rangle\left|e_{n}, \mathcal{S} \mathscr{A}\right\rangle, \\
\left|e_{n}, \mathcal{S} \mathscr{A}\right\rangle & \equiv \widehat{U}_{\left.\mathcal{S} \mathscr{A}\right|_{\mathscr{P}}}\left(e_{n}, t\right)|\mathcal{S} \mathscr{A}, t=0\rangle,
\end{aligned}
$$

it is easy to prove that $\left\{\left|e_{n}, \mathcal{S} \mathscr{A}\right\rangle\right\}$ forms an orthonormal basis (This is true, e.g., if $\left|e_{n}, \delta \mathscr{A}\right\rangle$ is the eigenstate of $\widehat{H}_{\left.\mathcal{S A}\right|_{\mathscr{P}}}\left(e_{n}, t\right)$ with eigenvalue $\left.E_{\left.\mathcal{S A}\right|_{\mathscr{P}}}\left(e_{n}, t\right)\right)$ and

$$
\widehat{U}_{\mathscr{P}}(t)|\mathscr{P}, t=0\rangle=\sum_{r=0}^{D_{\mathscr{P}}-1} g_{r}\left|e_{n}, \mathscr{P}\right\rangle .
$$

Equation (14) looks as if the single system $\mathscr{P}$ brings the properties $\left\{g_{r}^{2},\left|e_{n}, \mathscr{P}\right\rangle\right\}$ of the whole $\mathscr{P}-\mathcal{S} \mathscr{A}$ system. This is of course not true as the Schmidt basis is the joint properties of the whole system. The specific form of $\widehat{U}_{\mathscr{P}}(t)|\mathscr{P}, t=0\rangle$ stems from the specific choice of $\widehat{e}_{\mathscr{P}}$ in the Schmidt basis of $|\mathscr{P}(\mathcal{S} \mathscr{A}), t\rangle$. Actually, in this case, we can choose $\widehat{e}_{\mathscr{P}}=\rho_{\mathscr{P}}$.

When applying the ICQT to quantum gravity coupled with matter quantum fields [41], the usual Schrödinger equation losses its dynamical meaning and becomes trivially a constraint $\widehat{H}_{\mathscr{P} \mathscr{A} \mathscr{A}}|\mathscr{P}(\mathcal{S} \mathscr{A})\rangle=0$, known as the Wheeler-DeWitt equation. In this case, the programming observable $\widehat{e}_{\mathscr{P}}$ then corresponds the Dirac observable [27, 42]; dynamics described by a pair of the Schrödinger equations can be recovered due to the dual entanglement structure. Therefore, in this field-theoretical case, the significance and necessity of the information-complete dynamics in trinity is physically more transparent. Moreover, if $\mathscr{P}$ is the quantized gravitational field, then the Hamiltonians $\widehat{H}_{\mathcal{S}}, \widehat{H}_{\mathscr{A}}$, and $\widehat{H}_{\mathcal{S} \mathscr{A}}$ are simply ruled out as the gravitational field universally couples with any form of matter.

To end this section, it is important to note that the distinguished roles of $\mathscr{P}$ and $\mathcal{S} \mathscr{A}$ are relative. Depending on the specific form of the trinary Hamiltonian, we could have another possibility that $\delta \mathscr{A}$ can programme the evolution of $\mathscr{P}$. In this case, the programming basis is chosen for $\mathcal{S} \mathscr{A}$ and associated with an observable being commutative with $\widehat{H}_{\mathcal{S} \mathscr{A}}$ such that the $\mathscr{P}-\mathcal{S} \mathscr{A}$ measurability and a similar dynamics as in equation (9) can still be obtained. Furthermore, the roles of $\mathscr{P}$ and $\mathcal{S} \mathscr{A}$ are actually symmetric due to a nice property of the Schmidt decomposition [43], in which if an orthonormal basis labelled by an index (e.g., $n$ ) is chosen for a system, then the orthonormal basis for another system, by acting a unitary transformation upon it, is labelled by the same index. This property implies that both of the bases are already the Schmidt bases, up to local unitary transformations. A similar consideration is applicable to the programmed measurability $\left.\mathcal{S} \mathscr{A}\right|_{\mathscr{P}}$, too.

\section{Relation with Conventional Quantum Theory}

What is the relation between the ICQT and the usual QT? Before answering this question, first of all, we have to ask ourselves: Why are we bothered to revise the conventional QT into the current formulation of such a strange appearance? Here we must introduce at the very beginning the interacting/entangling $\mathscr{P}+\mathcal{S} \mathscr{A}$ trinity with a dynamical evolution (being always unitary) determined by the information-completeness principle such that the physical properties of parameters, e.g., $\mathscr{P}$ and $\mathcal{S} \mathscr{A}$, are coherently and completely stored in the $\mathscr{P}-\mathcal{S} \mathscr{A}$ entanglement and can only be predicted conditionally on each other. Anyway, in nonrelativistic quantum mechanics, there seems to be no physical motivation to introduce $\mathscr{P}$. This is in sharp contrast to traditional QT, where the " $\mathcal{S}+\mathscr{A}$ " description is sufficient and isolated, and single systems (free particles, free quantum fields, and so on) can have certain physical properties which can be accessed by a mysterious and nonunitary measurement process.

However, one of the most important lessons learned from general relativity is that spacetime is dynamical and the same thing as gravity. Therefore, there are, even in principle, no perfectly isolated systems as they must live in and couple with dynamical spacetime. If we quantize everything of nature, even gravity (spacetime), which mechanism could trigger a nonunitary measurement process? Of course, one could simply ignore, as an approximation, the dynamical and quantum nature of spacetime as the common wisdom does. Then, why such an ignorance could be a safe approximation without causing any internal inconsistency or incompleteness of traditional QT? In any case, spacetime is such an elementary physical entity. Anyone who does not shut eyes to these problems, among other interpretational difficulties, has to conclude that traditional QT must be incomplete as far as spacetime is not treated physically as a quantum system; the information-completeness principle is a possible remedy to complete current quantum formalism, as we suggest.

Ultimately, we should describe nature with quantum field theory. An information-complete quantum field theory [41] can indeed be formulated; the ICQT developed here for finite-dimensional quantum mechanical systems is thus the conceptual preparation and the mathematical formulation for that purpose. Therein, if we regard $\delta$ as matter fermion fields and $\mathscr{A}$ as their gauge fields, and $\mathcal{S} \mathscr{A}$ together as matter fields, then we immediately recognize that system $\mathscr{P}$ must be the gravitational field (i.e., spacetime), nothing else, as only gravitational field, while self-interacting, universally interacts with all other fields. Recall that in our $\mathscr{P}+\mathcal{S} \mathscr{A}$ trinity, $\mathscr{P}$ must universally couple with $\mathcal{S}$ and $\mathscr{A}$. If we think this way, an amazing picture (Figure 1) of our world arises: the gravitational field and matter fields are mutually defined and entangled-no matter, no gravity (spacetime), and vice versa-and for each of their entangled patterns, matter 
fermion fields and their gauge fields are likewise mutually defined and entangled. If this is indeed what our nature works to obey the information-completeness principle guaranteeing the completeness of the theory from the outset, the conventional QT will be an approximation of our ICQT when we ignore quantum effects of nature's programming system, i.e., gravity. Under such an approximation, the ICQT reduces to conventional QT, characterized by the usual Schrödinger equation and the probability description, and as such, QT in its current form is thus informationincomplete. This is in the exact sense that classical Newtonian mechanics is an approximate theory of special relativity when a physical system has a speed much less than the speed of light.

On the contrary, no matter how weak gravity is, it is forced to be there by the information-completeness principle, to play a role for completing a consistent quantum theory. This unique role of gravity (or spacetime) in our theory is consistent with the remarkable fact that only gravity is universally coupled to all matter fields. Of course, our current quantum description is an extremely good approximation. But for scales near the Planck one and for early Universe, quantized spacetime acts as the programming system and the ICQT will be necessary. Thus, both facts (i.e., current QT works so well, and quantum gravity effects are so weak at normal scales) hide so deeply any new theoretical architecture, like the ICQT, beyond current QT. Even in the string theory and loop quantum gravity, there is no change of the underlying quantum structure. By contrast, what we suggest within the ICQT is that at the level of quantized fields including quantized spacetime, everything is quantized and one does not have the usual separation of quantum systems and observers. In this case, one has to give up the classical-quantum hybrid feature of current QT. For this purpose, the most obvious way seems to be the elimination of the measurement postulate in our fully quantum (namely, not classical-quantum hybrid) description of nature. As we hope to argue, giving up the classical concepts associated with the measurement postulate in current QT does not lead to any sacrifice of our predictive power as the complete information is encoded by the dual entanglement structure.

If we take the above argument seriously, then the ICQT captures the most remarkable trinity of nature, namely, the division of nature by matter fermions, their gauge fields, and gravity (spacetime), though the role of the Higgs field needs a separate consideration (see Ref. [44] on this issue). The previous two sections argued the necessity of the information-completeness in the trinary description. Here we see that it is also sufficient: we do not have to invoke more programming systems to program $\mathscr{P} \mathcal{S} \mathscr{A}$ simply because we do not have spacetime out of spacetime-Trinity is necessary and sufficient. This eliminates the von Neumann chain in the usual quantum measurement model, in which one could introduce more other quantum systems interacting with the trinity.

One of the most challenging problems in current physics is how to put QT and general relativity into a single, consistent theory. To achieve this, it is encouraging to have a quantum formalism like the ICQT, in which gravity must be quantized and plays an essential role. As we showed elsewhere [41], following the above arguments indeed leads to a consistent quantum framework of unifying spacetime (gravity) and matter, without the fundamental inconsistencies [42] between gravity and conventional quantum field theory, implying the conceptual advantages of our theory. For instance, with the theoretical input from loop quantum gravity predicting the quantized geometry [27, 42, 45-47], the information-complete quantum field theory naturally explains the holographic principle, as well as its generalization, via spacetime-matter entanglement. As we currently understand it, the holographic principle [48-50] imposes a strong limit on the allowed states of quantum system in any finite spacetime regime. Such a limit paves the way to escape the infrared and ultraviolet singularities (divergences) that occur in conventional quantum field theory [27, 42].

Thus, the ICQT gives a strong motivation or reason for quantizing spacetime/gravity; there is no trinity if there is no quantized gravity. The natural position of gravity in the ICQT cannot be accidental and may be a strong evidence supporting our information-complete description of nature. It is surprise to see that nature singles out gravity as a programming field, which plays a role that is definitely different from matter fields. However, quantizing gravity as yet another field, as in conventional quantum field theory, is not sufficient and does not automatically result in a correct and consistent quantum theory of all known forces. Only when the information-completeness in the trinary description is integrated into our quantum formulation, can we have the desired theory of the Universe. The distinct roles of matter-matter (matter fermions and their gauge fields) entanglement and spacetime-matter (i.e., gravity-matter) entanglement indicate the reason why quantizing gravity as usual quantized fields suffers from well-known conceptual problems.

As an abstract mathematical structure, the current QT is content irrelevant in the following sense: while it is believed to be universally applicable to physical systems of any physical contents, ranging from elementary particles and (super)strings to the whole Universe, what physical content that it describes does not matter, and the physical content never changes its very structure. The situation for classical mechanics is quite similar in this aspect. However, the ICQT changes this in a dramatic way in the sense that the trinary picture of nature has to be integrated into a consistent formulation to enable an information-complete description. The physical content that the ICQT describes does matter as the states and their dynamical evolution of the trinary system are constrained or structured into the dual forms specified above. In particular, the inclusion of the programming system, identified with gravity in the field-theoretical case, is very essential and necessary in our description.

To end this section, let us briefly consider further conceptual applications of the ICQT. For informationcomplete quantum systems, interactions lead to $\mathscr{P}-\mathcal{S A}$ entanglement and the programmed $\mathcal{S}-\mathscr{A}$ entanglement from a separable initial state. This might hint the possibility that the Universe as a whole has an increasing entanglement, 
a kind of entanglement arrow of time (see also Ref. [41]). We note related analysis on the role of entanglement in the thermodynamic arrow of time in the framework of conventional [51, 52] or time-neutral formulation [53] of quantum mechanics. As gravity arguably plays an essential role in our information-complete description of nature, it is intriguing to see that gravity plays some role in the occurrence of the second law of thermodynamics and the arrow of time, as hinted in the study of black-hole thermodynamics [42, 54-56]. In the Diósi-Penrose model $[57,58]$, gravity was argued to play certain role for the wave function collapse.

Moreover, the conceptual difficulty of applying usual QT to the whole Universe disappears in our ICQT. Actually, the ICQT is a self-explaining quantum structure and does not need observers for its own formulation. The Universe described by the ICQT is thus self-explaining: The constituent parts in trinity are mutually defining and measuring in a specific dual entanglement structure, eliminating any subjective aspects regarding the current interpretations of quantum states-the existence of the Universe does not rely on the existence of potential observers observing the Universe. Entanglement in the dual form encodes, without relying on any external observers, all physical information and can give all physical predictions of the theory.

\section{Information-Complete Quantum Computation}

A new theory should make new predictions or/and motivate new applications; for new predictions of our theory, see Refs. $[41,44]$. Of course, previous interpretations of QT are very important for a better understanding of the theory. However, no interpretations make new predictions or/and motivate fundamentally new applications. Now we argue that our ICQT indeed motivates new applications if we consider its computational power. Even though gravity would play certain role in our future understanding of nature, artificial information-complete quantum systems are realizable without quantizing gravity.

What is an information-complete quantum computer (ICQC)? We define the ICQC as an artificial informationcomplete quantum system, or a quantum intelligent system (qubeing), which has an information-complete trinary structure consisting of $\mathcal{S}, \mathscr{A}$, and $\mathscr{P}$. The ICQC starts from an initial state $|\mathrm{ICQC}\rangle_{0}=|\psi, \mathcal{S}\rangle|\phi, \mathscr{A}\rangle|\chi, \mathscr{P}\rangle$. As usual, the $\mathcal{S}$ system has $n$ qubits, and thus dimensions of $2^{n}$. To be well defined, we also use qubits to make up the $\mathscr{A}$ system and the $\mathscr{P}$ system; $\mathscr{A}(\mathscr{P})$ has $n_{\mathscr{A}}\left(n_{\mathscr{P}}\right)$ qubits and dimensions of $2^{n_{\mathscr{A}}}\left(2^{n_{\mathscr{P}}}\right)$. To satisfy the information-completeness principle, we have $n_{\mathscr{A}}=n$ and $n_{\mathscr{P}}=2 n$. Our ICQC then works by applying certain patterns of quantum logic gates (singlequbit, two-qubit, and three-qubit gates), determined by quantum algorithm pertaining to the question under study. Generally speaking, as an artificially controllable quantum system, the patterns of gates are allowed to exhaust all unitary operations on the whole $\mathscr{P} \mathcal{S} \mathscr{A}$ system. Here, for our purpose, we consider a simplified ICQC; namely, we only perform the programmed measurement operation $\widehat{U}_{\mathscr{P}(\mathcal{S} \mathscr{A})}$ on $\mathscr{P} \mathscr{A}$. The resulting final state of the ICQC reads $|\mathrm{ICQC}\rangle=\widehat{U}_{\mathscr{P}(\mathscr{S A})}|\mathrm{ICQC}\rangle_{0}$ with

$$
\widehat{U}_{\mathscr{P}(\mathcal{S} \mathscr{A})}=\sum_{p=0}^{4^{n}-1}|p, \mathscr{P}\rangle\langle p, \mathscr{P}| \widehat{U}_{\mathscr{P}} \widehat{U}_{\mathscr{S A}}\left(\widehat{s}_{p}, \widehat{\mathbf{z}}\right),
$$

where the pair observables are defined by $\widehat{U}_{\mathcal{S A}}\left(\widehat{s}_{p}, \widehat{\mathbf{z}}\right)$ and, particularly, $\widehat{s}_{p}$ spans a complete operator set for $\mathcal{S}$, and $\widehat{\mathbf{z}}$ denotes $n$ Pauli's operators $\widehat{z}$ for $\mathscr{A}$.

Is the ICQC defined above a usual quantum computer merely with more $\left(n+n_{\mathscr{A}}+n_{\mathscr{P}}=4 n\right)$ qubits, but without the information-completeness and trinary structure? The answer is definitely "no" because of the conceptual difference between the two quantum computing devices. To see this, we prepare each qubit of $\delta$ in the initial state $|+, \mathcal{\delta}\rangle=(1 / \sqrt{2})(|0, \mathcal{S}\rangle+|1, \mathcal{S}\rangle)$ such that $|\psi, \mathcal{S}\rangle$ is in a superposition of all $2^{n}$ bit values with equal probability amplitude: $|\psi, \mathcal{S}\rangle=\left(1 / \sqrt{2^{n}}\right) \sum_{x=0}^{2^{n}-1}|x, \mathcal{S}\rangle$. The initial states of $\mathscr{A}$ and $\mathscr{P}$ are likewise prepared: $|\phi, \mathscr{A}\rangle=\left(1 / \sqrt{2^{n}}\right) \sum_{\chi=0}^{2^{n}-1}|y, \mathscr{A}\rangle \quad$ and $|\chi, \mathscr{P}\rangle=\left(1 / \sqrt{4^{n}}\right) \sum_{z=0}^{x^{n}-1}|z, \mathscr{P}\rangle$. Such a coherent superposition of conventional quantum computer's initial states is believed to be the very reason for the speedup of quantum algorithms $[28,59]$.

In the present case of the simplified ICQC, $\widehat{U}_{\mathscr{P}(\mathcal{S A})}$ can encode information-complete programmed measurement operations upon $\mathscr{A}$ and $\mathcal{S}$. These operations actually determine the allowed quantum algorithms and their outputs (along the $z$-basis) on $n$-qubit state $|\psi, \delta\rangle$, in the terminology of conventional quantum computing, in which the usual quantum measurement (also along the $z$-basis) is now replaced by the programmed entanglement created by $\widehat{U}_{\mathcal{S A}}\left(\widehat{s}_{p}, \widehat{\mathbf{z}}\right)$. Then we immediately see that in the ICQC, one has dual parallelism: parallelism in initial states as usual and parallelism of programmed operations (algorithms and outputs). In other words, a single ICQC with 4n qubits could compute in parallel $2^{2 n}$ algorithms of usual quantum computers with $n$ qubits. Due to this particular dual parallelism enabled by the ICQC, it is reasonable to expect much higher computational power with the ICQC.

Actually, the ICQC is, by definition, the most powerful computational machine on qubit systems in the sense of information-completeness; otherwise it is information-incomplete. Finding algorithms on the ICQC to explicitly demonstrate the computational power of the ICQC is surely a future interesting problem. Also, computational complexity and error tolerance in the ICQC framework are two important issues. If nature does use the information-completeness as a guiding principle, it computes the world we currently know (see [41] for a further discussion); such a world could be simulated and thus comprehensible by the ICQC in principle.

\section{Conclusions and Outlook}

In the present work, we have presented an interpretationfree QT under the assumption that quantum states of physical systems represent an information-complete code of any possible information that one might access. To make the 
information-completeness explicit in our formalism, the trinary picture of describing physical systems seems to be necessary. Physical systems in trinity evaluate and are entangled both in a dual form; quantum entanglement plays a central role in the ICQT-our world is information given in terms of entanglement at the most fundamental level. So the ICQT modifies two postulates (on quantum states and on dynamics) of current quantum mechanics in a fundamental way and eliminates the measurement postulate from our description; as a result of the modifications, the observables can be either information-complete (for $\mathscr{P}$ or $\mathcal{S A}$ ) or information-incomplete (for $\mathcal{S}$ or $\mathscr{A}$ ). We give various evidence and conceptual applications of the ICQT, to argue that the ICQT, naturally identifying gravity as nature's programming system in the field-theoretic case, might be a candidate theory capable of unifying matter and gravity (spacetime) in an information-complete quantum framework; for further development on our theory in the context of quantum gravity coupled with matter, see Ref. [41]. In this sense, the conventional QT will be an approximation of our ICQT when quantum effect of gravity is ignored. Such an approximation leads to the approximate Schrödinger equation and the probability description of current QT. This is in the exact sense that classical Newtonian mechanics is an approximate description of relativistic systems. The ICQT motivates an interesting application to information-complete quantum computing.

As we argued above, the current quantum mechanics is not information-complete because of its classical-quantum hybrid feature and, thus, suffers from interpretational difficulties. The explicit demand of information-completeness not only removes the conceptual problem of our current understanding of quantum mechanics, but also leads to a profound constraint on formulating quantum theory. Thus, the ICQT should not be understood simply as another interpretation of current QT; rather, it, by giving up the classical concept of probability associated with the measurement postulate, generalizes current quantum formalism-the physical prediction (outcomes of an observable and the corresponding probabilities) of a quantum measurement in conventional QT is now entailed by entanglement; no entanglement implies no information and thus no prediction. As we noted previously, adding informationcompleteness requirement into our current quantum formalism leads to serious consequences: information-completeness not only restricts the way on how to describe physical systems, but also the way how they interact/entangle with each other [44]. This will thus give a very strong constraint on what physical processes could have happened or be allowed to happen.

On the one hand, the ICQT provides a coherent conceptual picture of, or sheds new light on, understanding some problems or phenomena in current physics, including the intrinsic trinity of matter fermions, gauge fields and gravity, the arrow of time, and the holographic principle. On the other hand, some other problems, such as the complementarity principle, quantum nonlocality [25], and quantum communication, should be reconsidered from the viewpoint of the ICQT. All current quantum communication protocols $[28,60,61]$ have to make use of classical concepts on information. It is thus very interesting to see how to do communication in the ICQT and, particularly, to see whether or not it is possible to achieve unconditionally secure communication.

According to the ICQT, the world underlying us is all about information (entanglement); it is information-complete, deterministic, self-defining, and thus objective. Such a world view ("quantum determinism") is of course quite different from what we learn from current quantum mechanics, but in some sense, it returns to Einstein's world view and, not surprisingly, represents an embodiment of Wheeler's thesis known as "it from bit" [62]. Such a viewpoint calls for a reconsideration of our current understanding on physical reality, information, spacetime (gravity), and matter, as well as their links. Let us cite the famous Einstein-Podolsky-Rosen paper [1] here: "While we have thus shown the wave function does not provide a complete description of the physical reality, we left open the question of whether or not such a description exists. We believe, however, that such a theory is possible." It is too early to judge whether or not our ICQT completes current quantum mechanics in the Einstein-Podolsky-Rosen sense cited above, as experiments will be the ultimate judgement. But if nature does work like a description provided by the ICQT, nature will be very funny, and more importantly, nature does be comprehensible via a self-defining quantum structure. Einstein might be very happy to see that two of his important theoretical achievements, namely, general relativity (after being quantized in modern language) and the concept of quantum entanglement (discovered by him, together with Podolsky and Rosen), are very essential for our information-complete quantum description.

\section{Data Availability}

This work does not have any experimental data.

\section{Disclosure}

The draft [63] of the current work was posted on arXiv as https://arXiv:1412.1079.

\section{Conflicts of Interest}

The author declares that he has no conflicts of interest.

\section{Acknowledgments}

The author is grateful to Chang-Pu Sun for bringing Refs. $[25,37]$ into his attention and to Xian-Hui Chen, Dong-Lai Feng, and Yao Fu for enjoyable discussions. The author also acknowledges University of Science and Technology of China, where the work was initiated.

\section{References}

[1] A. Einstein, B. Podolsky, and N. Rosen, "Can quantummechanical description of physical reality be considered complete?" Physical Review, vol. 47, no. 10, pp. 777-780, 1935. 
[2] N. Bohr, "Can quantum-mechanical description of physical reality be considered complete?" Physical Review, vol. 48, no. 8, pp. 696-702, 1935.

[3] J. A. Wheeler and W. H. Zurek, Eds., Quantum Theory and Measurement, Princeton University Press, Princeton, NJ, USA, 1983.

[4] R. Omnès, "The interpretation of quantum mechanics," Princeton University Press, Princeton, NJ, USA, 1994.

[5] W. H. Zurek, "Decoherence, einselection, and the quantum origins of the classical," Reviews of Modern Physics, vol. 75, no. 3, pp. 715-775, 2003.

[6] M. Schlosshauer, "Decoherence, the measurement problem, and interpretations of quantum mechanics," Reviews of Modern Physics, vol. 76, p. 1267, 2004.

[7] C.-P. Sun, "Quantum dynamical model for wave-function reduction in classical and macroscopic limits," Physical Review A, vol. 48, no. 2, pp. 898-906, 1993.

[8] C.-P. Sun, X.-X. Yi, and X.-J. Liu, "Quantum dynamical approach of wavefunction collapse in measurement process and its application to quantum Zeno effect," Fortschritte der Physik/Progress of Physics, vol. 43, no. 7, pp. 585-612, 1995.

[9] A. E. Allahverdyan, R. Balian, and T. M. Nieuwenhuizen, "Understanding quantum measurement from the solution of dynamical models," Physics Reports, vol. 525, no. 1, pp. 1-166, 2013.

[10] G. L. Long, "General quantum interference principle and duality computer," Communications in Theoretical Physics, vol. 45, p. 825, 2006.

[11] G. L. Long, W. Qin, Z. Yang, and J.-L. Li, "Realistic interpretation of quantum mechanics and encounter-delayedchoice experiment," Science China: Physics, Mechanics and Astronomy, vol. 61, Article ID 30311, 2018.

[12] M. F. Pusey, J. Barrett, and T. Rudolph, "On the reality of the quantum state," Nature Physics, vol. 8, no. 6, pp. 475-478, 2012.

[13] G. C. Ghirardi and R. Romano, "On the completeness of quantum mechanics and the interpretation of the state vector," Journal of Physics: Conference Series, vol. 442, Article ID 012002, 2013.

[14] S. Aaronson, "Get real," Nature Physics, vol. 8, no. 6, pp. 443-444, 2012.

[15] J. Conway and S. Kochen, "The free will theorem," Foundations of Physics, vol. 36, no. 10, pp. 1441-1473, 2006.

[16] J. S. Bell, Speakable and Unspeakable in Quantum Mechanics, Cambridge University Press, Cambridge, UK, 1987.

[17] J. F. Clauser and M. A. Horne, "Experimental consequences of objective local theories," Physical Review D, vol. 10, no. 2, pp. 526-535, 1974.

[18] T. Scheidl, R. Ursin, J. Kofler et al., "Violation of local realism with freedom of choice," Proceedings of the National Academy of Sciences, vol. 107, no. 46, pp. 19708-19713, 2010.

[19] J. S. Bell, "On the Einstein Podolsky Rosen paradox," Physics, Long Island, NY, USA, 1964.

[20] J. von Neumann, Mathematical Foundations of Quantum Mechanics, Princeton University Press, Princeton, NJ, USA, 1996.

[21] E. P. Wigner, "The problem of measurement," American Journal of Physics, vol. 31, no. 1, pp. 6-15, 1963.

[22] L. D. Landau and E. M. Lifshitz, Quantum Mechanics: NonRelativistic Theory, Butterworth-Heinemann, Oxford, UK, 3rd edition, 1981.

[23] D. Bohm, "A suggested interpretation of the quantum theory in terms of "hidden" variables. I," Physical Review, vol. 85, no. 2, pp. 166-179, 1952.

[24] H. Everett III, “"Relative state" formulation of quantum mechanics," Reviews of Modern Physics, vol. 29, no. 3, pp. 454-462, 1957.
[25] F. J. Tipler, "Quantum nonlocality does not exist," Proceedings of the National Academy of Sciences, vol. 111, Article ID 11281, 2014.

[26] C. Rovelli, "Relational quantum mechanics," International Journal of Theoretical Physics, vol. 35, no. 8, pp. 1637-1678, 1996.

[27] C. Rovelli, Quantum Gravity, Cambridge University Press, Cambridge, UK, 2004.

[28] M. A. Nielsen and I. L. Chuang, Quantum Computation and Quantum Information, Cambridge University Press, Cambridge, UK, 2000.

[29] L. Hardy, https://arxiv.org/abs/0101012v4, 2001.

[30] C. A. Fuchs, https://arxiv.org/abs/0205039, 2002.

[31] M. Pawłowski, T. Paterek, D. Kaszlikowski, V. Scarani, A. Winter, and M. Żukowski, "Information causality as a physical principle," Nature, vol. 461, p. 1101, 2009.

[32] R. Colbeck and R. Renner, "No extension of quantum theory can have improved predictive power," Nature Communications, vol. 2, no. 1, p. 411, 2011.

[33] C. H. Bennett, H. J. Bernstein, S. Popescu, and B. Schumacher, "Concentrating partial entanglement by local operations," Physical Review A, vol. 53, no. 4, pp. 2046-2052, 1996.

[34] R. T. Thew, K. Nemoto, A. G. White, and W. J. Munro, "Qudit quantum-state tomography," Physical Review A, vol. 66, Article ID 012303, 2002.

[35] P. Busch, "Informationally complete sets of physical quantities," International Journal of Theoretical Physics, vol. 30, no. 9, pp. 1217-1227, 1991.

[36] J. M. Renes, R. Blume-Kohout, A. J. Scott, and C. M. Caves, "Symmetric informationally complete quantum measurements," Journal of Mathematical Physics, vol. 45, no. 6, pp. 2171-2180, 2004.

[37] S.-W. Li, C. Y. Cai, X. F. Liu, and C. P. Sun, "Objectivity in quantum measurement," Foundations of Physics, vol. 48, no. 6, pp. 654-667, 2018.

[38] S. Popescu and D. Rohrlich, "Thermodynamics and the measure of entanglement," Physical Review A, vol. 56, no. 5, pp. R3319-R3321, 1997.

[39] V. B. Braginsky, Y. I. Vorontsov, and K. S. Thorne, "Quantum nondemolition measurements," Science, vol. 209, no. 4456, pp. 547-557, 1980.

[40] V. B. Braginsky and F. Y. Khalili, "Quantum nondemolition measurements: the route from toys to tools," Reviews of Modern Physics, vol. 68, no. 1, pp. 1-11, 1996.

[41] Z.-B. Chen: 2014, https://arxiv.org/abs/1412.3662.

[42] T. Thiemann, "Lectures on loop quantum gravity," Quantum Gravity, vol. 631, pp. 41-135, 2003.

[43] J. Audretsch, Entangled Systems: New Directions in Quantum Physics, pp. 149-150, Wiley-VCH Verlag, Weinheim, Germany, 2007.

[44] Z.-B. Chen: 2016, https://arxiv.org/abs/1611.02662.

[45] C. Rovelli and L. Smolin, "Discreteness of area and volume in quantum gravity," Nuclear Physics B, vol. 442, no. 3, pp. 593-619, 1995.

[46] C. Rovelli, "Black hole entropy from loop quantum gravity," Physical Review Letters, vol. 77, no. 16, pp. 3288-3291, 1996.

[47] A. Ashtekar, J. Baez, A. Corichi, and K. Krasnov, "Quantum geometry and black hole entropy," Physical Review Letters, vol. 80, no. 5, pp. 904-907, 1998.

[48] G. 't Hooft, https://arxiv.org/abs/9310026, 1993.

[49] L. Susskind and J. Math, "The world as a hologram," Journal of Mathematical Physics, vol. 36, no. 11, pp. 6377-6396, 1995. 
[50] R. Bousso, "The holographic principle," Reviews of Modern Physics, vol. 74, no. 3, pp. 825-874, 2002.

[51] L. Maccone, "Quantum solution to the arrow-of-time dilemma," Physical Review Letters, vol. 103, Article ID 080401, 2009.

[52] D. Jennings and T. Rudolph, "Entanglement and the thermodynamic arrow of time," Physical Review E-Statistical Physics, Plasmas, Fluids, and Related Interdisciplinary Topics, vol. 81, Article ID 061130, 2010.

[53] J. B. Hartle, The Quantum Mechanical Arrows of Time, in Fundamental Aspects of Quantum Theory: a Two-Time Winner, D. Struppa and J. Tollaksen, Eds., Springer, Heidelberg, Germany, 2013.

[54] J. D. Bekenstein, "Black holes and the second law," Lettere Al Nuovo Cimento Series 2, vol. 4, no. 15, pp. 737-740, 1972.

[55] J. D. Bekenstein, "Black holes and entropy," Physical Review D, vol. 7, p. 2333, 1993.

[56] S. W. Hawking, "Black hole explosions?" Nature, vol. 248, no. 5443 , pp. $30-31,1974$.

[57] L. Diósi, "A universal master equation for the gravitational violation of quantum mechanics," Physics Letters A, vol. 120, p. 377, 1987.

[58] R. Penrose, "On Gravity's role in quantum state reduction," General Relativity and Gravitation, vol. 28, no. 5, pp. 581-600, 1996.

[59] S. Aaronson, "The limits of quantum computers," Scientific American, vol. 298, no. 3, p. 62, 2008.

[60] C. H. Bennett and G. Brassard in Proceedings of the IEEE International Conference on Computers, Systems and Singal, p. 175, Bangalore, India, December 1984.

[61] C. H. Bennett, G. Brassard, C. Crépeau, R. Jozsa, A. Peres, and W. K. Wootters, "Teleporting an unknown quantum state via dual classical and Einstein-Podolsky-Rosen channels," Physical Review Letters, vol. 73, p. 3081, 1993.

[62] J. A. Wheeler, "Recent thinking about the nature of the physical world: it from bit," Annals of the New York Academy of Sciences, vol. 655, pp. 349-364, 1992.

[63] Z.-B. Chen: 2014, https://arxiv.org/abs/1412.1079. 